\title{
Motion Rehab 3D AVE V2: um novo VR-exergame para fisioterapia motora
}

\section{Motion Rehab 3D AVE V2: a new VR-exergame for motor physical therapy}

\author{
Jean Marcel Vergouwen \\ University of Passo Fundo \\ Passo Fundo, RS, Brazil \\ 152169@upf.br \\ André Costa Pellin \\ University of Passo Fundo \\ Passo Fundo, RS, Brazil \\ 173702@upf.br
}

\author{
Nícolas Enrique de Quadros \\ University of Passo Fundo \\ Passo Fundo, RS, Brazil \\ 172985@upf.br \\ Mateus Trombetta \\ University of Passo Fundo \\ Passo Fundo, RS, Brazil \\ trombetta.mateus@gmail.com \\ Rafael Rieder \\ University of Passo Fundo \\ Passo Fundo, RS, Brazil \\ rieder@upf.br
}

\author{
Renato Vargas Júnior \\ University of Passo Fundo \\ Passo Fundo, RS, Brazil \\ 166496@upf.br
}

Patrícia Paula Bazzanello Henrique

University of Passo Fundo

Passo Fundo, RS, Brazil

patriciabazzanello@hotmail.com

\begin{abstract}
Resumo - Este artigo apresenta o desenvolvimento de uma nova versão do jogo Motion Rehab AVE 3D, exergame com suporte a Realidade Virtual que auxilia na reabilitação motora de membros superiores e inferiores. $O$ jogo orienta na execução de uma série de exercícios por parte do paciente, e pode ser configurado pelo profissional de saúde. Por intermédio de elementos visuais e sonoros, e imersão no ambiente virtual, pode incentivar o paciente durante seu processo de reabilitação. Esse trabalho destaca a implementação de recursos para suportar um novo sensor de movimentos e um novo head-mounted display, bem como a criação de novos cenários e a definição de novos exercícios, com orientação de fisioterapeutas.
\end{abstract} Unity.

Palavras-chave-Exergame, Reabilitação, Realidade Virtual,

Abstract - This paper presents the development of a new version of the Motion Rehab AVE 3D, an exergame supporting Virtual Reality to help the motor rehabilitation of upper and lower limbs. The game guides the patient to execute a series of exercises, and a physician can configure the sequence. Considering visual and sound elements, and immersion in the virtual environment, it can engage the patient during his/her rehabilitation process. This work highlights the development of resources to support a new motion sensor and a new headmounted display, as well as the creation of new scenarios and the definition of new exercises, with guidance from physiotherapists.

Index Terms-Exergame, Rehabilitation, Unity, Virtual Reality.

\section{INTRODUÇÃO}

Exergames são aplicações computacionais que combinam a dinâmica de jogo com exercícios, permitindo às pessoas a prática de atividades físicas, tanto para o lazer, como para a reabilitação em saúde [1][2]. Eles são capazes de capturar, por exemplo, os movimentos naturais de um paciente e, assim, promover uma interação física. Eles funcionam como espelho virtual, pois auxiliam o paciente na percepção dos movimentos e podem prover feedback visual do que está ocorrendo [3] [4] [5]. O uso de dispositivos de interação torna o tratamento mais encorajador, estimulando os sentidos humanos (principalmente, visão e audição e, alguns casos, o tato).

Nesse contexto, o projeto do exergame Motion Rehab tem uma relação direta com o uso de exergames na fisioterapia. Originalmente desenvolvido por Trombetta et al. [6], o jogo oferece níveis de atividades destinados à reabilitação motora de membro superior e equilíbrio de indivíduos que sofreram Acidente Vascular Encefálico. Ele combina serious game e Realidade Virtual (RV) como ferramenta para o processo de reabilitação de movimentos de pessoas, de maneira interativa e explorando a percepção espacial, com temáticas que ajudam para que a recuperação de um paciente seja mais descontraída e intuitiva.

O presente artigo apresenta a continuidade de desenvolvimento do jogo Motion Rehab AVE 3D [7], descrevendo o andamento da nova versão do jogo, considerando novos recursos computacionais e exercícios de fisioterapia para reabilitação motora de pacientes com acompanhamento profissional. $\mathrm{O}$ trabalho considera as etapas de fundamentação e estudo de ferramentas, a criação de dois novos cenários virtuais imersivos, o suporte a dois novos dispositivos (um de visualização, e outro de rastreamento), e a definição dos novos exercícios a serem implementados, com apoio constante de profissionais de saúde.

Para tanto, este trabalho está assim organizado: a Seção 2 faz a apresentação do jogo original, a Seção 3 apresenta os materiais e métodos utilizados na criação de novos cenários e suporte a novos dispositivos, a Seção 4 apresenta o trabalho em andamento com recursos incorporados para a nova versão até o momento, e a Seção 5 mostra as conclusões e os trabalhos futuros.

\section{MOTION REHAB}

O Motion Rehab AVE 3D é um exergame desenvolvido com a game engine Unity, e faz uso de dispositivos para captação de movimentos e visualização em RV para proporcionar uma ferramenta de apoio ao processo de reabilitação convencional. O projeto tem intenção de oferecer essa ferramenta com um custo benefício viável para ser utilizada pelos profissionais de saúde. 
O exergame propõe atividades em que o paciente realiza uma série de movimentos considerando objetos e desafios disponíveis em um cenário virtual. O jogo contempla, atualmente, seis atividades que consideram exercícios de flexão, abdução, adução do ombro, abdução e adução horizontal do ombro, extensão de cotovelo, extensão de punho, flexão de joelho, flexão e abdução de quadril em um espaço 3D (Figura 1). As atividades consideram exercícios usados em sessões de reabilitação convencionais e foram definidas com ajuda de uma fisioterapeuta.

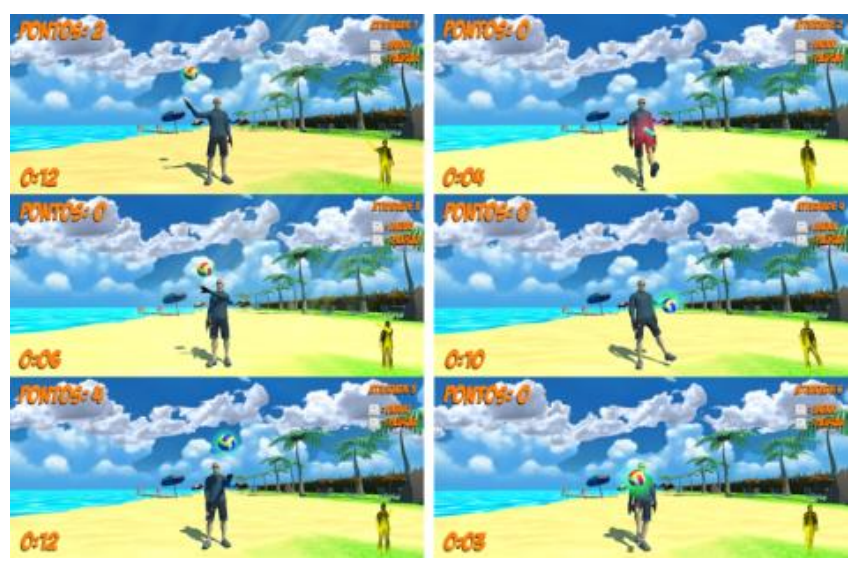

Figura 1. Mosaico apresentando os níveis de jogo atualmente disponíveis noMotion Rehab AVE 3D [6].

É apresentado para o usuário profissional de saúde, ao iniciar o jogo, um menu para selecionar uma fase, dificuldade e um avatar para representar o paciente. Ao iniciar uma fase, o jogador (paciente) pode observar seu personagem representado seus movimentos. No topo da tela, as informações de pontuação, tempo restante e dificuldade são exibidas.

O projeto Motion Rehab, desenvolvido em 2015, foi criado originalmente com uma interface $2 \mathrm{D}$ com suporte ao sensor de movimentos Kinect v1. Na versão atual, o jogo possui uma interface $3 \mathrm{D}$ e tem suporte ao sensor de movimentos Kinect v2 e ao dispositivo de visualização e rastreamento Oculus Rift (modelos DK1 e DK2). Um módulo adicional (Motion Rehab 3D Plus [8]) pode ser acionado pelo fisioterapeuta para criar um programa de atividades personalizado a seus pacientes (Figura 2), para atendimento individualizado e com coleta dos dados de desempenho ao longo do processo de reabilitação.

Sabe-se que existem diferentes estudos relacionados à utilização de exergames em processos de reabilitação de membros superiores e inferiores, que empregam o monitoramento de movimentos dos indivíduos por meio de sensores [9][10]. Como exemplo, pode-se destacar os estudos de Norouzi-Gheidari et al. [11], Passos et al. [12], Choi e Paik [13], e González-González et al. [14], que utilizaram exergames para a reabilitação de pacientes que sofreram acidente vascular encefálico, sendo que cada abordagem considerou aspectos físicos distintos.

Apesar desses estudos cumprirem o propósito de auxiliar na terapia convencional e motivar a realização dos exercícios, o problema é que cada um deles apresenta uma solução distinta, que atende somente um propósito específico. Isso acarreta, pelo menos, dois empecilhos para o profissional da saúde que deseja utilizar exergames em sua clínica: o alto custo de aquisição de diversos equipamentos e programas de computador; e o aprendizado para utilizar cada um deles.

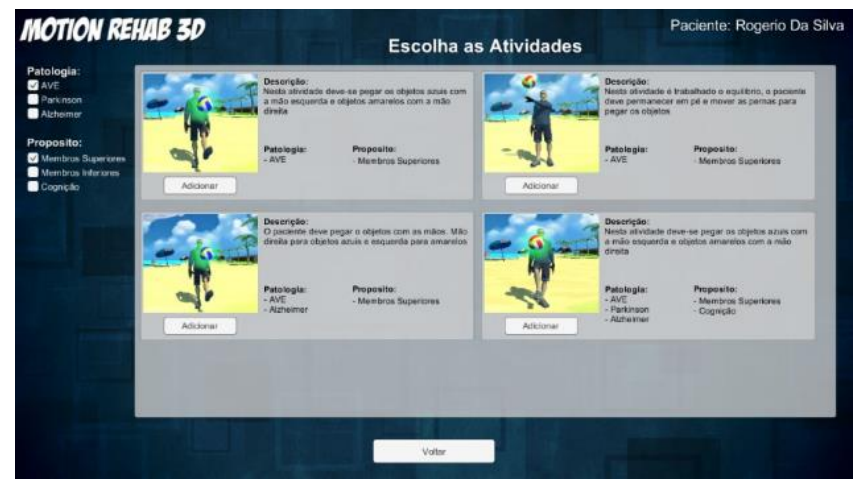

Figura 2. Interface do módulo Motion Rehab 3D Plus para seleção de atividades [8].

É nesse contexto que a continuidade do exergame Motion Rehab AVE 3D se justifica, uma vez que o objetivo é inserir novos níveis de jogos em uma solução já existente. Além disso, oferecer variadas tarefas de reabilitação recorrentes em clínicas, disponibilizando ao profissional de saúde uma ferramenta que auxilie o tratamento personalizado para um grupo variado de pacientes.

Para tanto, foi discutido sobre a importância de incluir novos cenários motivadores e imersivos, capazes de agregar outras atividades de recuperação para pacientes, bem como dar suporte a novos dispositivos de interação.

Com isso em mente, foram criados dois novos ambientes virtuais, incluindo-se o mapeamento de outros exercícios (recomendados por fisioterapeuta) e equipamentos mais recentes. Os ambientes virtuais contemplam a representação de uma área de hidroterapia e um espaço de praça pública. Em relação aos exercícios, recebeu-se orientação do profissional de saúde para monitorar e mapear exercícios de agachamento, abdução horizontal de ombro, abdução horizontal de membro superior e abdução e adução de membro inferior. A próxima seção aborda isto em detalhes.

\section{MATERIAIS E MÉTODOS}

Nos projetos desenvolvidos para o exergame Motion Rehab são utilizados diferentes ferramentas que permitem a modelagens dos objetos e cenários, o desenvolvimento e programação dos jogos, e o suporte a dispositivos imersivos e de captura de movimentos. As próximas subseções explicam os materiais e métodos utilizados no projeto da nova versão do jogo.

\section{A. Plataformas de desenvolvimento}

Para este projeto, adotou-se a Unity como game engine para o desenvolvimento dos níveis de jogo do Motion Rehab. Ela é uma das plataformas de desenvolvimento de jogos $2 \mathrm{D}$ e 3D mais conhecidas, tem vasta documentação e possibilita a programação em alto nível ao desenvolvedor [15].

Já para a criação dos cenários e objetos do jogo, optou-se pelo 3ds Max. Esse programa de modelagem tridimensional permite também renderização de imagens e animações [16], bem como a exportação dos modelos para o formato FBX, já utilizado na versão atual do jogo.

\section{B. Dispositivos de visualização e rastreamento}

Como o exergame Motion Rehab é um jogo 3D, e também pode ser jogado de maneira imersiva, é necessário suporte a alguns dispositivos de visualização e rastreamento. Eles têm a função de fazer com que o usuário tenha uma interação completa com o jogo, causando uma sensação de presença 
durante o processo interativo. Com esses dispositivos podem ser usadas algumas técnicas que estimulam os sentidos sensoriais nos seus usuários [17].

Um dos principais equipamentos para que a pessoa consiga ter uma experiência imersiva é o uso do headmounted display (HMD). Este dispositivo tem a função de transmitir duas imagens, uma para cada olho, através de displays óticos e controle da razão de aspecto, além de rastrear os movimentos de cabeça. As imagens são combinadas pelo cérebro (da mesma forma que percebemos o ambiente real com as imagens captadas por nossos olhos), o que proporciona uma ilusão de profundidade em nossa visão, uma técnica que é chamada de estereoscopia. Atualmente, o jogo tem suporte aos modelos Rift DK1 e DK2 [18]. No entanto, existem versões mais recentes de HMDs, como o Oculus Quest [18] e o Vive [19].

Para a captura dos movimentos do corpo do jogador durante o processo interativo no exergame, pode-se utilizer do mapeamento realizado por sensores de movimentos. Geralmente, esses equipamentos são compostos de uma câmera RGB para reconhecimento facial, um sensor de profundidade para mapear o ambiente, um microfone embutido para captação de ruídos, e uma API de reconhecimento de pontos de articulação do corpo humano. Atualmente, o jogo tem suporte aos modelos Kinect Xbox 360 (V1) e One (V2).

\section{DESENVOLVIMENTO}

As próximas subseções apresentam o trabalho em andamento da nova versão do jogo, denominada Motion Rehab 3D AVE V2.

\section{A. Suporte a novo sensor de movimentos}

Anteriormente, o Motion Rehab foi desenvolvido com suporte aos sensores de movimentos Kinect V1 e Kinect V2. Entretanto, devido a descontinuidade destes sensores pela Microsoft, foi preciso buscar um novo dispositivo para fazer a substituição.

Duas opções foram consideradas, com o intuito de reduzir ou eliminar a dependência com APIs de sensores de movimento, e considerando os equipamentos disponíveis para a pesquisa em laboratório: utilizar a biblioteca OpenPose [20] (dispensando o uso de sensores de movimentos, e dependendo apenas de uma câmera RGB), e/ou utilizar a biblioteca Nuitrack SDK [21], que tem suporte a diferentes sensores de movimento (entre eles, o Orbbec Astra [22] e os próprios Kinect V1 e V2).

Ao avaliar a biblioteca OpenPose, dois problemas foram encontrados: a incapacidade de capturar profundidade (impedindo a atribuição de movimentos em três dimensões), e o alto poder computacional preciso para sua execução.

Em relação a biblioteca Nuitrack SDK, foi possível incorporá-la facilmente ao projeto e realizar a captura de movimentos. Ela dispõe de uma versão gratuita (três minutos de interação) e uma versão paga, com preço acessível (interação ilimitada), além de boa documentação para projetos que envolvem sensores de movimento.

Nesse contexto, trabalhou-se também na inclusão de suporte ao sensor de movimentos Orbbec Astra PRO, escolhido para substituir o Kinect. Ele possibilita atribuir facilmente os movimentos capturados para um avatar 3D, além de ter um desempenho superior ao do Kinect, ser portátil e também ter um custo acessível (comparado a outros modelos de mercado). Os únicos contras são a ineficiência de capturar pequenos detalhes de profundidade e alcance curto, similar ao Kinect. Porém esses requisitos são irrelevantes para esta versão do jogo, pois os exercícios são mapeados para o movimento de pacientes em espaços curtos, utilizando HMD.

\section{B. Suporte a novo head-mounted display}

Conforme abordado anteriormente, o Motion Rehab AVE 3D também possui suporte para visualização em terceira pessoa, sem a necessidade de um HMD. Porém, para a experiência imersiva, e para melhorar a eficiência da reabilitação, o projeto foi reconfigurado para também dar suporte ao HTC Vive (além do Rift).

Nos primeiros testes somente com o uso do HTC Vive (HMD e controles), o jogo comportou-se como o esperado na execução de tarefas em atividades que não requerem a captura de movimentos espaciais. Porém, num segundo teste preliminar, com todo o conjunto de dispositivos, notou-se que existe um conflito entre os sensores de HTC Vive e do Orbbec Astra PRO. Para tanto, testes mais apurados estão sendo projetados para avaliar se o conflito tem relação com o mapeamento de movimentos em software, ou se é um problema em relação aos sensores de profundidade existentes nos dois equipamentos.

\section{Criação de novos cenários}

Foram definidos novos cenários 3D para a nova versão do exergame, considerando exercícios de fisioterapia que exploram a percepção espacial, estabelecidos com a orientação da fisioterapeuta. Os novos cenários virtuais representam uma área de hidroterapia e uma praça pública, conforme Figura 3 e Figura 4.

A escolha destes ambientes se deu por conta de serem ambientes reais onde as pessoas em geral estão acostumadas a praticar atividades de fisioterapia (piscina e acessórios na hidroterapia) e exercícios que estimulam a habilidade motora (academias ao ar livre na praça).

Além disso, considerou-se estudos que destacam que ambientes ricos em estímulos influenciam positivamente no aumento da estimulação sensorial, cognitiva e motora [23]. A percepção do ambiente a nossa volta pode influenciar diretamente na evolução do tratamento [24]. Diante disso, os elementos criados para compor os cenários propostos consideram características que transmitem conhecimentos ergonômicos adequados e habituais para um ambiente terapêutico.

Para a criação dos cenários virtuais, foi iniciado um estudo para entender o funcionamento dos ambientes, para que as criações artísticas representassem da melhor forma a realidade desses espaços. Após levantamento dos dimensionamentos adotados nesses espaços para o uso real, foram definidas dimensões para a piscina, mobiliários da área de hidroterapia, e elementos identificadores de uma praça pública, incorporando-os aos cenários virtuais.

Após a concretização dos cenários, fez-se a exportação das malhas e dos mapas de texturas para o projeto na Unity, utilizando conversão de modelos 3D para o formato FBX. Em projeto, ainda foram realizados ajustes de iluminação e testes de renderização das faces, para a exibição correta dos ambientes em tempo de execução. 


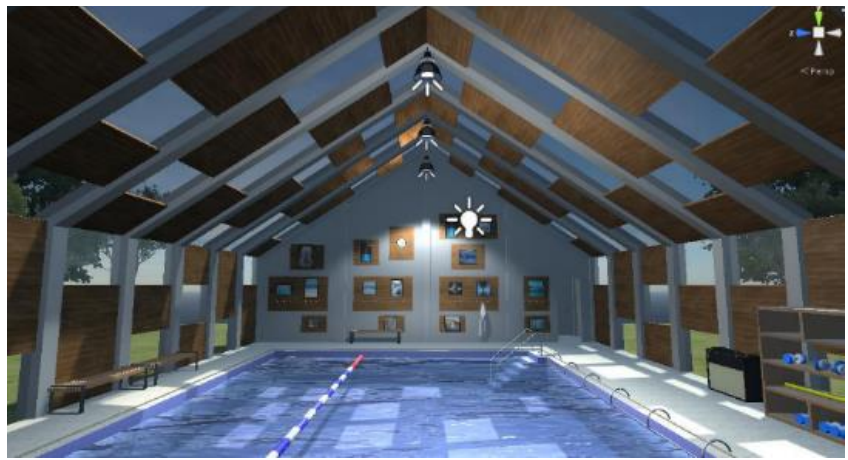

Figura 3. Visão de novo cenário de jogo: piscina de hidroginástica.

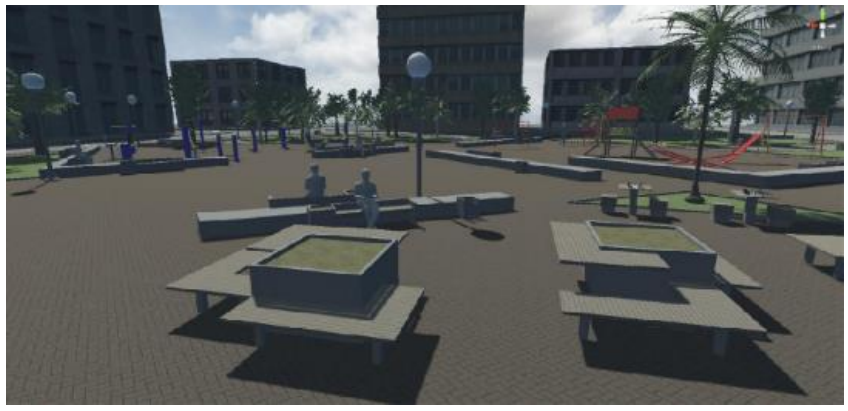

Figura 4. Visão de novo cenário de jogo: praça pública.

\section{Definição de novos exercícios}

Os novos exercícios propostos pela fisioterapeuta para a nova versão do exergame Motion Rehab consideram o agachamento, a abdução horizontal de ombro, a abdução horizontal de membro superior e a abdução e adução de membro inferior (Figura 5).

Este projeto decidiu por tais exercícios cinesioterapêuticos pois os mesmos priorizam a funcionalidade e permitem que os usuários os realizem de forma agradável e eficaz. São exercícios que envolvem grandes grupos musculares e podem melhorar a resistência, o equilíbrio e aumentar a funcionalidade para as atividades de vida diária, proporcionando ganhos no aspecto social, mental e físico, contribuindo também para maior autonomia e qualidade de vida [25].

No cenário de hidroterapia serão mapeados pelo jogo os exercícios de agachamento e de abdução horizontal de ombro, enquanto no cenário da praça pública serão considerados os exercícios de abdução horizontal de membro superior e de abdução e adução de membro inferior.

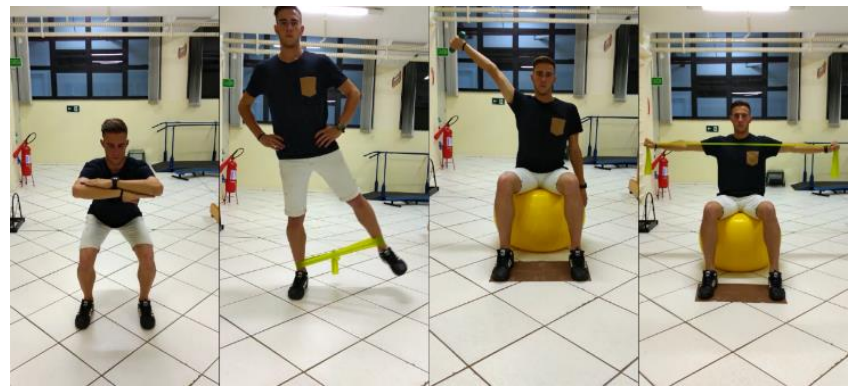

Figura 5. Representação dos novos exercícios incorporados aos cenários da piscina e da praça.

Esta etapa ainda está em fase de desenvolvimento. Para a definição de gameplays das tarefas do jogo (relacionadas ao monitoramento da execução dos exercícios propostos), o projeto adotará a aplicação da metodologia ágil Scrum [26], considerando sprints, definidos e revisados em sprint plannings e sprint reviews com a fisioterapeuta.

\section{CONCLUSÃO E TRABALHOS FUTUROS}

Este trabalho apresentou o andamento do desenvolvimento do VR-exergame Motion Rehab 3D AVE V2, uma nova versão do jogo Motion Rehab AVE 3D. Foram apresentados os novos cenários e exercícios concebidos para o jogo, bem como o suporte aos novos dispositivos de visualização e rastreamento.

Os exergames estão cada vez mais ganhando espaço na área da saúde, e a geração desse jogo pode ser uma ferramenta útil para ajudar fisioterapeutas na reabilitação motora e de equilíbrio de seus pacientes, independente do acometimento.

Os próximos objetivos do projeto estão relacionados a resolução do conflito entre o HMD e o sensor de movimentos, bem como a definição de gameplays para os novos exercícios dentro da dinâmica do jogo. Após isto, prevê-se uma fase de testes de rastreamento dos movimentos, com acompanhamento da fisioterapeuta, para validar o mapeamento e as ações do jogador.

Por último, será realizada avaliação com pacientes de duas clínicas de fisioterapia para analisar a usabilidade e a utilidade do jogo, considerando tarefas de visualização e rastreamento em primeira pessoa. Para tanto, prevê-se a submissão prévia do projeto ao Comitê de Ética em Pesquisa, para obter aprovação do protocolo de realização dos experimentos com usuários e profissionais de saúde.

\section{AGRADECIMENTOS}

Este trabalho faz parte do escopo do projeto "Motion Rehab AVE 3D: um exergame de Realidade Virtual para reabilitação pós-AVE", Chamada Universal MCTIC/CNPq

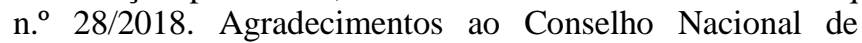
Desenvolvimento Científico e Tecnológico (CNPq) pelo auxílio financeiro e de cotas de iniciação científica, e para a Fundação Universidade de Passo Fundo (FUPF) pela concessão de bolsas PIBIC/CNPq e PIBIC/UPF.

\section{REFERÊNCIAS}

[1] Huang, H. et al. How to create flow experience in exergames? Perspective of flow theory. Telematics and Informatics, v. 35, n. 5, p. 1288-1296, 2018

[2] Lyons, E. J. Cultivating engagement and enjoyment in exergames using feedback, challenge, and rewards. Games for health journal, v. 4, n. 1 , p. 12-18, 2015.

[3] Taylor, M.J.; Lindsverk, H.; Nygaard, M.; Hunt, J.; Shanks, J.; Griffin, M.; Shawis, T.; Impson, R. Comparing the energy expenditure of Wii $\neg$ Fit $\neg$ based therapy with that of traditional physiotherapy in an older adult population. J Am Geriatr Soc., v.62, n.1, p.203-205, 2014.

[4] Anderson, F.; Annett, M.; Bischof, W.F. Lean on Wii: physical rehabilitation with virtual reality Wii peripherals. Stud Health Technol Inform., v. 154, p. 229-234, 2010.

[5] Bao, X.; Mao, Y.; Lin, Q.; Qiu, Y.; Chen, S.; Li, L.; Cates, R. S.; Zhou, S.; Huang, D. Mechanism of Kinect $\neg$ based virtual reality training for motor functional recovery of upper limbs after subacute stroke. Neural Regen Res., v. 5, n. 8, p. 2904-ᄀ13, 2013.

[6] Trombetta, M. et al. Motion Rehab AVE 3D: A VR-based exergame for post-stroke rehabilitation. Computer methods and programs in biomedicine, v. 151, p. 15-20, 2017.

[7] Trombetta, M. et al. Motion Rehab AVE 3D. 2016. Patente: Programa de Computador. Número do registro: BR512016001373-7, data de registro: 21/10/2016, título: "Motion Rehab AVE 3D" , Instituição de registro: INPI - Instituto Nacional da Propriedade Industrial.

[8] Trombetta, M. et al. Motion Rehab 3D Plus: Um Exergame Customizável Aplicado à Reabilitação Física. In: Simpósio Brasileiro 
de Computação Aplicada à Saúde (SBCAS), 2018, Natal. Anais do SBCAS, 2018. v. 18. p. 1-10.

[9] Lee, H. S.; Park, Y. J.; Park, S. W. The effects of virtual reality training on function in chronic stroke patients: a systematic review and metaanalysis. BioMed Research International, v. 2019, 2019.

[10] Aguilar-Lazcano, C. A. et al. Interaction modalities used on serious games for upper limb rehabilitation: a systematic review. Games for health journal, v. 8, n. 5, p. 313-325, 2019.

[11] Norouzi-Gheidari, N. et al. Interactive virtual reality game-based rehabilitation for stroke patients. In: 2013 International Conference on Virtual Rehabilitation (ICVR). IEEE, 2013. p. 220-221.

[12] Passos, N. R. S. et al. Siirius Surfer utilizando jogos serios na reabilitação de tronco para pacientes pós-AVC. Proceedings do XII Simpósio Brasileiro de Jogos e Entretenimento Digital (SBGames 2013), p. 25-28, 2013.

[13] Choi, Y. H.; Paik, N. J. Mobile game-based virtual reality program for upper extremity stroke rehabilitation. JoVE (Journal of Visualized Experiments), n. 133, p. e56241, 2018.

[14] González-González, C. S. et al. Serious games for rehabilitation: Gestural interaction in personalized gamified exercises through a recommender system. Journal of biomedical informatics, v. 97, p. 103266, 2019.

[15] Unity. Unity (game engine). Disponível em: <https://unity.com/>. Acesso: 25 jun. 2020.

[16] 3ds Max. 3ds Max: 3D Modeling, Animation and Rendering Software. Autodesk, Inc. Disponível em: <https://www.autodesk.com.br/products/3ds-max/overview>. Acesso: 25 jun. 2020.
[17] Burdea, Grigore C.; Coiffet, Philippe. Virtual reality technology. John Wiley \& Sons, 2003.

[18] Oculus. Oculus: equipamentos e headsets de realidade virtual. Disponível em: <https://www.oculus.com>. Acesso: 25 jun. 2020.

[19] Vive. Vive: Discover Virtual Reality Beyond Imagination. Disponível em: <https://www.vive.com/sea/>. Acesso: 25 jun. 2020.

[20] OpenPose. OpenPose: Real-time multi-person keypoint detection library for body, face, hands, and foot estimation. Disponível em: <https://github.com/CMU-Perceptual-Computing-Lab/openpose>. Acesso: 25 jun. 2020.

[21] Nuitrack. Nuitrack Full Body Skeletal Tracking Software - Kinect replacement for Android, Windows, Linux, iOS, Intel RealSense, Orbbec. Disponível em: 〈https://nuitrack.com>. Acesso: 25 jun. 2020.

[22] Orbbec. Orbbec: Intelligent computing for everyone everywhere. Disponível em: < https://orbbec3d.com>. Acesso: 25 jun. 2020.

[23] Arakawa, A.; Kaneko, M.; Narukawa, M. An investigation of factors contributing to higher levels of placebo response in clinical trials in neuropathic pain: a systematic review and meta-analysis. Clinical drug investigation, v. 35, n. 2, p. 67-81, 2015.

[24] Da Fonseca, L. J. P.; Brandalize, M.; Brandalize, D. Nintendo WII na reabilitação de pacientes com paralisia cerebral-relato de caso. Arquivos de Ciências da Saúde da UNIPAR, v. 16, n. 1, 2012.

[25] Vilela-Junio, J. F.; Soares, V. M. G.; Maciel, A. M. S. B. A importância pratica da cinesioterapia em grupo na qualidade de vida de idosos. Acta Fisiátrica, v. 24, n. 3, p. 133-137, 2017.

[26] Keith, C. Agile Game Development with Scrum. Addison-Wesley, 2010 . 